Revista de Psicología de la PUCP. Vol. XXII, 1, 2004

\title{
Sentimiento de sobrecarga y apoyo social en cuidadores familiares de enfermos crónicos ${ }^{1}$
}

\author{
Susana Seidmann ${ }^{2}$, Dorina Stefani ${ }^{3}$, Carlos O. Pano ${ }^{4}$, \\ Luisa Acrich ${ }^{5}$ y Vera Bail Pupko ${ }^{6}$ \\ Universidad de Buenos Aires
}

Se evalúa si la estructura de la red social, el apoyo percibido y su nivel de satisfacción personal inciden en el sentimiento de sobrecarga del cuidador, derivado de la asistencia de un hijo o de un progenitor anciano con una enfermedad crónica invalidante. Con tal fin, fueron administrados a 143 cuidadoras $-50,3 \%$ madres y $49,7 \%$ hijas- el Cuestionario sobre Apoyo Social de Mannheim (1986) y el Cuestionario sobre Sentimiento de Carga de Zarit y Zarit (1999). A partir de las pruebas multivariadas de significación estadística, se puede concluir que las madres presentan una red de parientes mayor que las hijas, aunque no se diferencian significativamente en la percepción del apoyo social. En ellas, el tamaño de la red de parientes, el apoyo instrumental cotidiano y psicológico en crisis aminora el sentimiento de sobrecarga, mientras que en las hijas lo disminuye la mayor intensidad de apoyo instrumental en crsis percibido por ellas.

Palabras clave: enfermedad crónica, cuidador familiar, apoyo social, sentimiento de sobrecarga.

\section{Feeling of burden and social support in family caregivers of chronically ill patients}

We evaluate if the social network structure, the perceived support and the personal satisfaction acts on the caregiver's feeling of burden, coming from the caring of a child or an older parent with chronic invalidating illness. We used Mannheim's Instrument for Social Support (1986) and the Feeling of Burden Questionnaire (Zarit \& Zarit, 1999) with 143 caregivers $-50,3 \%$ mothers and $49,7 \%$ daughters. Taking into account the multivariate statistical tests, we can conclude that mothers have a larger relatives network than daughters, even though they don't significantly differ in the perception of social support. In mothers, the size of the relatives network, the everyday instrumental and the psychological crisis support diminishes the feeling of burden, as for the daughters the more intense crisis instrumental perceived support diminishes the same feeling.

Key words: chronic illness, family caregivers, social support, feeling of burden.

Una versión del trabajo fue presentada en el XXVII Congreso Internacional de Psicología, Estocolmo, Suecia, 23-28 de julio de 2000. Este trabajo forma parte del proyecto de investigación TP 46, 1998-2000, subsidiado por la Secretaría de Ciencia y Técnica de la Universidad de Buenos Aires (UBACYT).

2 Doctora en Psicología, UBA. Profesora Adjunta Regular de Psicología Social, Investigadora de la Secretaría de Ciencia y Técnica (UBACYT) en el Instituto de Investigaciones de la Facultad de Psicología, Directora del Proyecto TP 46. Correo electrónico: seidmann@psi.uba.ar 

La enfermedad crónica de una persona es un acontecimiento de gran impacto que involucra a toda su familia. Se destaca especialmente la figura del cuidador familiar (Biegel, Sales \& Schulz, 1991), conformado en su gran mayoría por mujeres (Aguas, 1998, 1999). El cuidador familiar asume centralmente la responsabilidad del cuidado y la toma de decisiones respecto de la enfermedad de su familiar enfermo.

Según Rolland (1994), una enfermedad crónica implica un tiempo prolongado de padecimiento (más de 6 meses) que puede tener un comienzo agudo o insidioso, una evolución progresiva o cíclica y un desenlace fatal, que acorte la expectativa de vida o que no la altere si la enfermedad remite. Estas situaciones van a tener sus correlatos específicos en la demanda al cuidador familiar, produciendo en él diferentes niveles de estrés.

Sobre la base del modelo transaccional del estrés propuesto por Lazarus y Folkman (1980), se asume que algunas de las situaciones

3 Licenciada en Sociología, UBA. Miembro de la Carrera del Investigador Científico del Consejo Nacional de Investigaciones Científicas y Técnicas (CONICET) categoría Principal, en el Centro Interdisciplinario de Investigaciones en Psicología Matemática y Experimental (CIIPME). Profesora Asociada de Metodología de la Investigación, Universidad Argentina J. F. Kennedy. Correo electrónico: stefanid@fibertel.com.ar

4 Licenciado en Ciencias Matemáticas. Profesor Titular Regular de Estadística, UBA. Investigador de la Secretaría de Ciencia y Técnica de la UBA (UBACYT). Correo electrónico: cpano@psi.uba.ar

5 Licenciada en Ciencias de la Educación, UBA. Profesora Adjunta de Psicología Social. Investigadora de la Secretaría de Ciencia y Técnica (UBACYT) en el Instituto de Investigaciones de la Facultad de Psicología. Correo electrónico: lacrich@psi.uba.ar

- Psicóloga. Jefa de Trabajos Prácticos de Psicología Social, UBA. Investigadora de la Secretaría de Ciencia y Técnica (UBACYT) en el Instituto de Investigaciones de la Facultad de Psicología. Correo electrónico: verabail62@yahoo.com.ar 
que moderan el impacto de la enfermedad crónica en la familia en general y en el cuidador en particular son la estructura de la red social personal y el apoyo social percibido que de ella deriva.

La red social personal está constituida por la totalidad de los vínculos que conectan a una persona con su entorno y le facilitan la satisfacción de sus necesidades materiales y emocionales. Su estructura está caracterizada por el tamaño, la composición y la densidad de aquellos que la conforman. Por otra parte, la red social cumple diferentes funciones que determinan en el sujeto la percepción del apoyo social. Esta es una experiencia de subjetivización de los recursos de cuidado que brinda la red e implica una conexión social a través de interacciones de apoyo que contribuyen a atemperar los efectos nocivos del malestar producidos por la enfermedad (Hofboll \& Vaux, 1993).

Veiel (1986) define el concepto de apoyo social como una relación entre las necesidades individuales y los recursos provistos por la red social. El apoyo social es una función de la red social, es un fenómeno relacional.

Los elementos objetivos de la red, en la medida en que se acoplan a las necesidades de las personas, van a configurar diversas situaciones de apoyo social:

- apoyo instrumental: objetos materiales útiles que producen cambios en la situación externa de la persona.

- apoyo psicológico: respuestas a las necesidades psicosociales de contacto, intimidad y pertenencia.

Ambos tipos de apoyo pueden hacerse efectivos cotidianamente o en situaciones de crisis. Quedan así conformados cuatro tipos de apoyo social: 
- apoyo psicológico cotidiano

- apoyo psicológico en crisis

- apoyo instrumental cotidiano

- apoyo instrumental en crisis

El cuidador familiar, sometido a múltiples demandas de acuerdo a la gravedad de la enfermedad y a la relevancia de la discapacidad, siente una sobrecarga que, según Taussig (1999), está asociada, entre otros, al costo emocional de la pena, al desempeño de roles adicionales, al estrés creciente, a la ruptura de la rutina cotidiana y al peso económico que representa la enfermedad.

El sentimiento de sobrecarga frente a la enfermedad puede derivar, asimismo, de la existencia de sentimientos contradictorios y ambivalentes hacia el enfermo o hacia su enfermedad (Biegel, Sales \& Schulz, 1991).

\section{Objetivos}

Los propósitos de este trabajo son: a) comparar a dos grupos de cuidadoras familiares de enfermos crónicos (madres que cuidan hijos o hijas que cuidan a un progenitor) en 1) el tamaño de la red de parientes, 2) la intensidad del apoyo social percibido en las funciones de apoyo, 3) la satisfacción con dicho apoyo y 4) el sentimiento de sobrecarga, y b) evaluar en cada grupo de cuidadoras si 1) el tamaño de la red de parientes, 2) la intensidad del apoyo social percibido en las funciones de apoyo y 3) la satisfacción con respecto al mismo influyen sobre el sentimiento de sobrecarga que genera en el cuidador la situación de enfermedad crónica de un familiar. 
Susana Seidmann, Dorina Stefani, Carlos O. Pano, Luisa Acrich y Vera Bail Pupko

\section{Hipótesis}

A partir de todo lo expuesto y en respuesta a los objetivos propuestos fueron formuladas las siguientes hipótesis:

1. Las prescripciones sociales correspondientes al rol materno y al rol filial influyen sobre la composición de la red (parientes - no parientes), la intensidad de apoyo social percibido en las cuatro funciones de apoyo, la satisfacción con respecto al mismo y el sentimiento de sobrecarga.

2. El tamaño de la red de parientes, la intensidad del apoyo social percibido en las funciones de apoyo y la satisfacción con respecto al mismo moderan el sentimiento de sobrecarga, tanto en las madres como en las hijas cuidadoras.

\section{Metodología}

\section{Variables}

Variables independientes

1. Relación de parentesco con el enfermo (madres que cuidan hijos - hijas que cuidan a un progenitor)

2. Estructura de la red social personal: tamaño de la red de parientes

3. Intensidad del apoyo social

4. Satisfacción con el apoyo social

Variable dependiente

Sentimiento de sobrecarga 
Sentimiento de sobrecarga y apoyo social en cuidadores familiares...

\section{Instrumentos}

Fue construido un cuestionario de datos sociodemográficos básicos del cuidador familiar y del enfermo, en el que fueron, además, incluidas preguntas acerca de la situación de cuidado: tipo de enfermedad crónica del familiar, desde cuándo se lo asiste y cuántas horas por día durante toda la semana.

Por otra parte, fueron seleccionadas las siguientes pruebas psicosociales:

1. Cuestionario sobre Apoyo Social de Mannheim (MISS) (Veiel, 1986)

Esta prueba de tipo multidimensional recaba información en forma cualitativa y cuantitativa acerca de la estructura de la red social de un sujeto, del apoyo social con que un individuo percibe que cuenta en caso de necesidad y de las diferentes funciones de apoyo social que cumple la red: apoyo psicológico cotidiano, apoyo instrumental cotidiano, apoyo instrumental en crisis y apoyo psicológico en crisis.

El MISS es una prueba multidimensional integrada por 37 enunciados que evalúan un aspecto objetivo referido a las características estructurales de la red social personal (tamaño, composición, frecuencia de contactos) y un aspecto subjetivo referido a las necesidades y percepciones del sujeto acerca del apoyo actual y potencial con el que cree contar en diferentes situaciones, y la correspondencia entre ambas dimensiones.

Los coeficientes de correlación entre los ítemes y el análisis factorial del instrumento evidencian, respectivamente, su confiabilidad y validez de constructo (Sacchi \& Richaud de Minzi, 2002). 
Para este estudio, el tamaño de la red de parientes fue categorizado en pequeño, mediano y grande de la siguiente manera: las cuidadoras que se ubicaban debajo del percentil 25 en la variable porcentaje de parientes de la red, fueron consideradas que tenían una red de parientes pequeña; aquellas que se ubicaban entre el percentil 25 y el 75, una red de parientes mediana; y las que se ubicaban por encima del percentil 75 , una red de parientes grande. El mismo procedimiento fue utilizado para categorizar la intensidad de apoyo social percibido en general y en cada una de las cuatro funciones de apoyo social: psicológico cotidiano, instrumental cotidiano, instrumental en crisis y psicológico en crisis. En la variable porcentaje de parientes de la red percibido por las cuidadoras como recurso efectivo o potencial en caso de necesidad, aquellas que se ubicaban debajo del percentil 25 se las consideró que percibían un apoyo social leve; aquellas que se ubicaban entre el percentil 25 y el 75 , un apoyo social moderado, y las que se ubicaban por encima del percentil 75 , un apoyo social fuerte.

\section{Cuestionario sobre Sentimiento de Carga (Zarit \& Zarit, 1999)}

Este instrumento evalúa el grado con el cual los sujetos que cuidan a un enfermo perciben que sus responsabilidades tienen un efecto negativo sobre su salud, vida personal y social, finanzas y bienestar emocional (Zarit \& Zarit, 1982). Consta de 22 ítemes construidos según la técnica de escalamiento de estímulos de Likert. El sujeto responde a cada uno de ellos marcando una de las siguientes alternativas de respuesta: 1: Nunca, 2: Casi nunca, 3: A veces, 4: Frecuentemente y 5 : Casi siempre. El puntaje total del sujeto en el cuestionario se obtiene promediando los valores correspondientes a cada respuesta a través de todos los ítemes. Este valor permite ubicarlo en un punto del continuo correspondiente al sentimiento de sobrecarga que varía desde 1 hasta 5 .

Un bajo puntaje en la escala denota un leve sentimiento de sobrecarga, mientras que, un alto puntaje en la misma indica un fuerte sentimiento. 
Sentimiento de sobrecarga y apoyo social en cuidadores familiares...

Los estudios psicométricos realizados sobre el instrumento original (Gallagher et al., 1985; Zarit \& Zarit, 1982) y sobre la versión castellana del mismo (Taussig, 1999) en muestras de familiares cuidadores de enfermos crónicos de habla inglesa y de habla española, revelaron su validez y confiabilidad.

\section{Participantes}

Los instrumentos fueron administrados a 143 familiares cuidadoras de enfermos crónicos, de las cuales el 50,3\% eran madres que cuidaban a un hijo en situación de desvalimiento y el resto, hijas que cuidaban a un progenitor anciano.

La mayor proporción de cuidadoras respondió estar casada (madres: 59\%, hijas: 48\%). Proporciones similares de ambos grupos habían finalizado estudios primarios y secundarios (madres: 69\%, hijas: $75 \%)$.

Con respecto al período de tiempo que asiste al enfermo, el $50 \%$ de las madres y el $56 \%$ de las hijas lo vienen haciendo desde menos de dos años, el $37 \%$ de las madres y el 18\% de las hijas entre dos y cinco años, y el $26 \%$ de las madres y el $13 \%$ de las hijas desde más de cinco años. En cuanto al tipo de enfermedad, el $56 \%$ de las madres y el $60 \%$ de las hijas asistía a un familiar con alguna enfermedad que produce discapacidad progresiva y que altera el ciclo de vida (por ejemplo: esclerosis múltiple, enficema, artritis reumatoidea), mientras que el resto en proporciones similares asistían a enfermos con discapacidad progresiva y fatal (madres: $28 \%$, hijas: $33 \%$ ) o discapacidad gradual que no altera el ciclo de vida (madres: $16 \%$, hijas: $7 \%$ ).

Por último, con respecto a la cantidad de horas diarias de cuidado durante toda la semana, el $28 \%$ de las madres y el $30 \%$ de las hijas le dedicaban de 5 a 12 horas, el $34 \%$ de las madres y el $11 \%$ de las hijas, 
menos de cinco horas, y el $38 \%$ de las madres y el $59 \%$ de las hijas, más de doce horas por día durante toda la semana.

\section{Análisis estadístico}

Con el fin de evaluar si existen diferencias estadísticamente significativas entre los grupos de madres e hijas cuidadoras en el tamaño de la red de parientes consignado en el MISS y el puntaje promedio obtenido en el Cuestionario sobre Sentimiento de Carga, fueron calculadas pruebas de diferencias de medias aritméticas para muestras independientes. Asimismo, para estudiar si ambos grupos difieren en la intensidad y la satisfacción con las funciones de apoyo social fueron calculados coeficientes de $\chi^{2}$ como prueba de independencia estadística.

Por otra parte, para el grupo de madres y el de las hijas cuidadoras, considerados en forma separada, fueron realizados ocho ANOVAS de dos factores con frecuencias desiguales en las celdas (Winer, 1971) con el objeto de evaluar en los puntajes obtenidos en el Cuestionario sobre Sentimiento de Carga los efectos de la intensidad y de la satisfacción con cada función de apoyo y la interacción entre estos efectos simples.

Para efectuar los análisis estadísticos correspondientes fue utilizado el Statistical Package for Social Sciences (SPSS).

\section{Resultados}

Los resultados correspondientes al primer objetivo planteado en este estudio indicaron que:

1. El tamaño de la red de parientes del grupo de las madres se diferenció significativamente del grupo de las hijas. El porcentaje 
Sentimiento de sobrecarga y apoyo social en cuidadores familiares...

promedio de parientes señalado por las madres $(65 \%)$ fue mayor que el consignado por las hijas $(53 \%) .\left(z_{o}=3.25 ; p=.000\right)$.

2. Existe independencia estadística entre el tipo de cuidadora familiar (madre o hija) y la intensidad de apoyo social percibido en las cuatro funciones de apoyo, tal como lo indican los valores de $\chi^{2}$ que se presentan en el Cuadro 1. Es decir, que ambos grupos de cuidadoras no se diferencian significativamente en la percepción de la intensidad de apoyo social en ninguna de las funciones de apoyo social instrumental diario y en crisis, y psicológico diario $\mathrm{y}$ en crisis.

\section{Cuadro 1}

Valores de $\chi^{2}$ para madres vs. hijas cuidadoras

\begin{tabular}{llcccc}
\hline & & \multicolumn{4}{c}{ Funciones de apoyo } \\
\cline { 3 - 6 } & & $\begin{array}{c}\text { Apoyo } \\
\text { instrumental } \\
\text { en crisis }\end{array}$ & $\begin{array}{c}\text { Apoyo } \\
\text { instrumental } \\
\text { Intensidario }\end{array}$ & $\begin{array}{c}\text { Apoyo } \\
\text { psicológico } \\
\text { en crisis }\end{array}$ & $\begin{array}{c}\text { Apoyo } \\
\text { psicológico } \\
\text { diario }\end{array}$ \\
\hline & $\begin{array}{l}\text { Leve } \\
\text { Moderado } \\
\text { Fuerte }\end{array}$ & 1.26 & 0.36 & 0.09 & 0.09 \\
\hline
\end{tabular}

Con dos grados de libertad: no significativos.

En el Cuadro 2 se observa que tanto las madres como las hijas percibían en mayor porcentaje un apoyo social moderado y fuerte en las cuatro funciones de apoyo. 
Susana Seidmann, Dorina Stefani, Carlos O. Pano, Luisa Acrich y Vera Bail Pupko

\section{Cuadro 2}

Porcentajes de madres e hijas en las distintas intensidades de apoyo social percibido en las cuatro funciones de apoyo

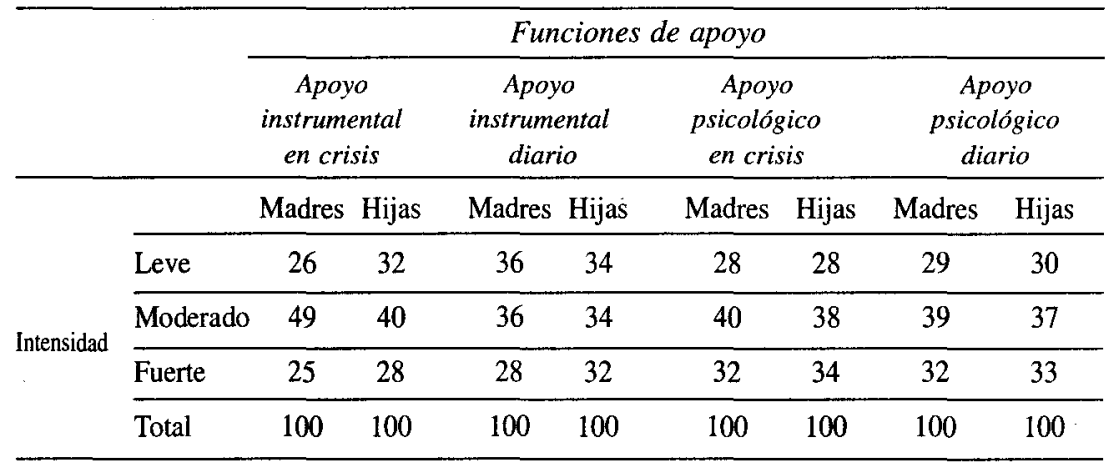

3. Las madres se diferenciaron significativamente de las hijas cuidadoras sólo en la satisfacción que manifestaron con el apoyo psicológico diario $\left(\chi_{(4,143)}^{2}=21.69 ; p=.000\right)$. Las madres estaban más satisfechas con las propuestas recibidas para realizar actividades concretas (madres: $31 \%$; hijas: $5 \%$ ); mientras que las hijas estaban más satisfechas con la comunicación de hechos cotidianos que les interesaba (madres: $11 \%$; hijas: $35 \%$ ).

4. Las madres y las hijas cuidadoras no se diferenciaron significativamente en el sentimiento de sobrecarga $\left(\mathrm{M}_{\text {madres }}=\right.$ $2.55 ; M_{\text {hijas }}=2.70 ; z_{o}=1.35$ ). Ambos grupos manifestaron en promedio sentirse "a veces" abrumada por el costo emocional que implicaba la enfermedad crónica de los miembros de su familia.

Los resultados obtenidos para cumplimentar el segundo objetivo mostraron en las cuidadoras familiares entrevistadas que:

1. El tamaño de la red de parientes influye significativamente sobre el sentimiento de sobrecarga $\left(\mathrm{F}_{(2,143)}=5.60 ; p=.004\right)$ tanto en las madres como en las hijas cuidadoras. Tal como se observa en el Cuadro 3, los puntajes promedios obtenidos por las cuidadoras entrevistadas en el Cuestionario sobre Sentimiento de Carga 
Sentimiento de sobrecarga y apoyo social en cuidadores familiares...

disminuye a medida que aumenta el tamaño de la red de parientes consignados por ellas.

\section{Cuadro 3}

Sentimiento de sobrecarga según tamaño de la red de parientes, en cuidadoras familiares de enfermos crónicos

\begin{tabular}{lcccc}
\hline & & \multicolumn{3}{c}{ Tamaño red de parientes } \\
\cline { 2 - 5 } & & Chico & Mediano & Grande \\
\hline $\begin{array}{l}\text { Sentimiento } \\
\text { de sobrecarga }\end{array}$ & $M$ & 2.88 & 2.54 & 2.36 \\
\cline { 2 - 5 } & $D E$ & .70 & .70 & .56 \\
\hline
\end{tabular}

2. De acuerdo a los valores $\mathrm{F}$ obtenidos al realizar los ANOVAS de dos factores correspondientes que se presentan en el Cuadro 4 , en el grupo de las madres cuidadoras el sentimiento de sobrecarga resultó influido por la satisfacción con respecto a los apoyos instrumental diario y psicológico en crisis, mientras que, en el de las hijas este sentimiento fue afectado por la intensidad del apoyo instrumental en crisis.

\section{Cuadro 4}

Funciones de apoyo social y sentimiento de sobrecarga en madres e hijas cuidadoras (ANOVA de dos factores)

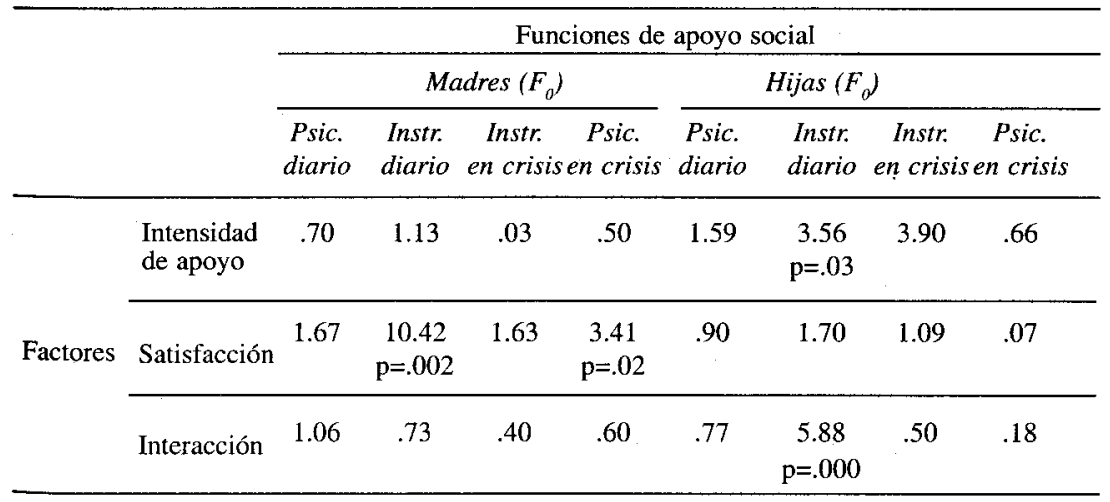


En el Cuadro 5 se observa que las madres que manifestaron estar satisfechas con los apoyos instrumental diario y/o psicológico en crisis expresaron un menor sentimiento de sobrecarga que aquellas que no estaban satisfechas. Por otra parte, este sentimiento es mayor en aquellas madres no satisfechas con el apoyo psicológico en crisis que en las que no lo estaban con el apoyo instrumental diario.

\section{Cuadro 5}

Sentimiento de sobrecarga según satisfacción con los apoyos instrumental diario y psicológico en crisis, en madres cuidadoras

\begin{tabular}{lcccccc}
\hline \multirow{2}{*}{ Satisfacción } & \multicolumn{2}{c}{$\begin{array}{c}\text { Apoyo instrumental } \\
\text { diario }\end{array}$} & & \multicolumn{2}{c}{$\begin{array}{c}\text { Apoyo psicológico } \\
\text { en crisis }\end{array}$} \\
\cline { 2 - 4 } \cline { 7 - 8 } & & No & Sí & & No & Sí \\
\cline { 2 - 4 } $\begin{array}{l}\text { Sentimiento de } \\
\text { sobrecarga }\end{array}$ & $M$ & 2.86 & .39 & & 3.22 & 2.30 \\
\cline { 2 - 4 } & $D E$ & .95 & .73 & & .84 & .80 \\
\hline
\end{tabular}

En el Cuadro 6 se observa que las hijas cuidadoras que perciben una mayor intensidad de apoyo instrumental en crisis manifestaron un menor sentimiento de sobrecarga.

\section{Cuadro 6}

Sentimiento de sobrecarga según intensidad de apoyo instrumental en crisis, en hijas cuidadoras

\begin{tabular}{lcccc}
\hline & & \multicolumn{3}{c}{ Intensidad del apoyo instrumental en crisis } \\
\cline { 2 - 5 } & & Leve & Moderado & Fuerte \\
\cline { 2 - 5 } Sentimiento & $M$ & 2.95 & 2.60 & 2.50 \\
\cline { 2 - 5 } de sobrecarga & $D E$ & .83 & .95 & .78 \\
\hline
\end{tabular}

Dado que en el grupo de las hijas cuidadoras existe una interacción significativa entre la intensidad de apoyo instrumental diario y la satisfacción con el mismo, en el Cuadro 7 se observa que en aquellas que perciben recibir un apoyo leve, el sentimiento de 
sobrecarga es menor en las que estaban satisfechas. Cuando la intensidad del apoyo es moderado o fuerte, el estar satisfecha o no satisfecha no afecta al sentimiento de sobrecarga.

\section{Cuadro 7}

Sentimiento de sobrecarga según intensidad y satisfacción con el apoyo instrumental diario, en hijas cuidadoras (promedios)

\begin{tabular}{lllcc}
\hline & & \multicolumn{4}{c}{ Apoyo instrumental diario } \\
\cline { 2 - 5 } & & \multicolumn{4}{c}{ Intensidad } \\
\cline { 2 - 5 } Satisfacción & No & 3.48 & 2.81 & 2.29 \\
\cline { 2 - 5 } & Sí & 2.46 & 2.90 & 2.58 \\
\hline
\end{tabular}

\section{Conclusiones}

Los resultados obtenidos en el estudio apoyan en parte las hipótesis planteadas. A partir de los mismos, se puede concluir, con un cierto margen de error, en las cuidadoras de familiares entrevistadas que:

- El tipo de cuidadora (madres o hijas) influye sobre la dimensión objetiva de la red social personal en cuanto al tamaño de la red de parientes y no parientes que les brindan apoyo frente a la situación de enfermedad crónica de un familiar. Las madres informaron contar con una red más amplia de parientes que las hijas.

Si bien para ambos grupos de cuidadoras, la red de parientes representa la fuente primaria de protección, la enfermedad de un niño o un joven involucra intensamente a toda la familia. Para ella, la enfermedad no representa sólo una situación inesperada, sino que además es considerada un hecho antinatural, inaceptable 
Susana Seidmann, Dorina Stefani, Carlos O. Pano, Luisa Acrich y Vera Bail Pupko 
Sentimiento de sobrecarga y apoyo social en cuidadores familiares...

Se seguirá profundizando el estudio sobre este tema para indagar más acerca de si las características negativas propias de la situación de cuidado de un familiar enfermo crónico explicarían este resultado.

Los resultados y conclusiones obtenidas en este trabajo completan las halladas en un estudio preliminar en el cual se llevó a cabo este análisis en las primeras 60 cuidadoras familiares entrevistadas (Stefani, Seidmann \& Acrich, 2000).

\section{Referencias}

Aguas, S. (1998). Análisis de los efectos experimentados por familiares cuidadores de adultos mayores con déficit en actividades de la vida diaria. Tesis de maestría no publicada. Universidad Autónoma de Madrid, Madrid, España.

Aguas, S. (1999). Una protección social invisible. Hechos y derechos, 6, 27-37.

Biegel, D. E., Sales, E. \& Schulz, R. (1991). Family caregiving in chronic illness. Newbury Park, CA: Sage Publications.

Hofboll, S. \& Vaux, A. (1993). Social support: Resources and context. En L. Goldberger \& S. Brenitz (Eds.), Handbook of stress: Theoretical and clinical aspects (pp. 685-705). Nueva York: Free Press.

Lazarus, R. S. \& Folkman, S. (1980). An analysis of coping in a middle-aged community sample. Journal of Health and Social Behavior, 21, 219-239.

Rolland, J. (1994). Family, illness and disability. Nueva York: Basic Books.

Sacchi, J. C. \& Richaud de Minzi, M. C. (2002). Adaptación argentina de la entrevista Manheim de apoyo social. Revista Interamericana de Psicología, 36(1-2), 127-148.

Stefani, D., Seidmann, S. \& Acrich, L. (2000). Estructura de la red social, apoyo social percibido y sentimiento de sobrecarga. Un 
estudio preliminar en cuidadoras de enfermos crónicos. Interdisciplinaria, Revista de Psicología y Ciencias Afines, 17(1), 21-38.

Taussig, I. M. (1999). Sentimientos de carga en cuidadores de enfermos de Alzheimer. En J. Buendía (Ed.). Familia y Psicología de la Salud (pp. 285-301). Madrid: Pirámide.

Veiel, H. (1986). Needs and networks. Manuscrito no publicado. Winer, B. J. (1971). Statistical principles in experimental design. Nueva York: McGraw Hill.

Zarit, J. M. \& Zarit, S. H. (1982, noviembre). Measurement of burden and social support. Documento presentado en la Reunión Científica Anual de la Gerontological Society of America, San Diego, CA, EE. UU.

Zarit, J. M. \& Zarit, S. H. (1999). Sentimientos de carga en cuidadores de Alzheimer (Cuestionario). En J. Buendía (Ed.), Familia y Psicología de la Salud. Madrid: Pirámide. 